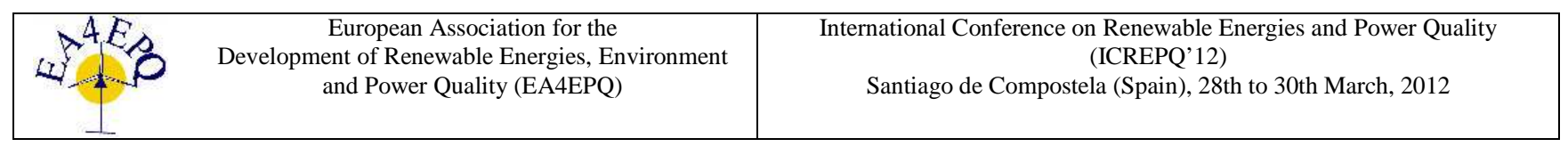

\title{
Controllogger: A remote monitoring system for decentralized renewable energy sources
}

\author{
Fábio T. Brito ${ }^{1}$, Sandro C.S. Jucá ${ }^{1}$ and Paulo C.M. Carvalho ${ }^{2}$ \\ ${ }^{1}$ Federal Institute of Education, Science and Technology of Ceará I.F.C.E \\ Campus Maracanaú - Ceará, 61925-315 Maracanaú (Brazil) \\ Phone/Fax number: 00558538786316 , e-mail: fabio@ifce.edu.br, sandrojuca@ifce.edu.br \\ ${ }^{2}$ Federal University of Ceará U.F.C \\ Campus Pici - Ceará, 60455-760 Fortaleza (Brazil) \\ Phone/Fax number: 0055853366 9585, e-mail: carvalho@dee.ufc.br
}

\begin{abstract}
The development of a remote monitoring and control system for decentralized renewable energy plants is described in the present paper. The system has a microcontroller-based unit for local storing and data transmission to internet by a GSM modem and a database server to store the data. The proposed architecture is easily extended for controlling a decentralized renewable energy plant by a remote operator. Correlations and performance tests are used to compare the data collected by the system prototype with data collected by a commercial data acquisition system. The results indicate that the Root Mean Square Error (RMSE) is $0.103 \mathrm{~m} / \mathrm{s}$ for the wind speed measurement and the correlation factor is greater than 0.9996 . Overall, the low errors rates indicate that the data have been stored in a similar form to what would be expected with the use of a commercial data logger.
\end{abstract}

\section{Key words}

Renewable Energy, Acquisition System, Microcontroller, Monitoring.

\section{Introduction}

The contribution of renewable energy plants in the world electricity production is increasing rapidly. In this way, data acquisition systems are essential to estimate the potential of renewable energy sources. For instance, a large quantity of data from different years is necessary to estimate scenarios using renewable energy sources. These aspects have an importance mainly for developing countries, where decentralized power plants based on renewable sources are in some cases the best option for supplying electricity to rural areas. Nevertheless, the cost of commercial data acquisition systems is still a barrier for a greater dissemination of such systems in developing countries [1].

A local web server (on board) is constrained by lower memory limitations for storage of large data amounts.
Hence, remote operators of renewable energy plants connected to that local web server can view only limited data [2]. The usually applied data organization in text files is inefficient and a development of an automate Database is indispensable [3]. The monitoring system consists of a microcontroller-based unit to acquire interest signals, while the collected data are transmitted to a Database server by a GSM modem. The GSM standard extends the effectiveness of the system independently wherever the plants are placed, even far from the electrical distribution network and from the traditional and wired telecommunication systems. Due to the low cost and diffusion of the GSM devices, the transmission system is fairly cheap and it is expected to became cheaper and cheaper [3]. The collected data are further processed, stored in the disk and displayed on the web page using the PHP language. This method has the advantage of a rapid data acquisition system development and provides an easy-to-use graphical environment that permits system operators to process easily the collected data. The maintenance operator presence in a decentralized renewable energy plant should be as low as possible, considering the moderate value of the produced energy [3]. In this way, the purpose of the present paper is to allow such plants to be remotely monitored and controlled by a remote operator.

\section{Developed Remote monitoring system}

The developed system consists of a microcontrollerbased unit which converts the electric signal from an anemometer to a microcontroller PIC 18F2550. The sensor information is processed and later sent to a GSM modem every 10 min by using a RS-232 interface.

\section{A. Field data-acquisition system (Controllogger)}

The electric description of the developed field dataacquisition system is shown in Table I. 
Table I. - Electric Specifications of the developed system

\begin{tabular}{|c|c|}
\hline Signal & Electric Specifications \\
\hline RS-232 & Pins: Tx, Rx and Ground \\
\hline USB & Pins:+5 V, Ground, D+ and D- \\
\hline Analog & Resolution: 10 bits \\
\cline { 2 - 2 } Input & Maximum Input Impedance: $10 \mathrm{k} \Omega$ \\
\hline Digital & Input voltage: $5 \mathrm{~V}$ \\
\cline { 2 - 2 } Input & Input impedance: $1 \mathrm{k} \Omega$ \\
\hline Analog & Range: 0 to $5 \mathrm{~V}$ \\
\cline { 2 - 2 } Output & Maximum current: $500 \mathrm{~mA}$ \\
\hline Digital & Output voltage: $10 \mathrm{~V}$ \\
Output & Maximum current: $500 \mathrm{Ma}$ \\
\hline
\end{tabular}

The field data acquisition system is called Controllogger; the electric board is shown in figure 1 .

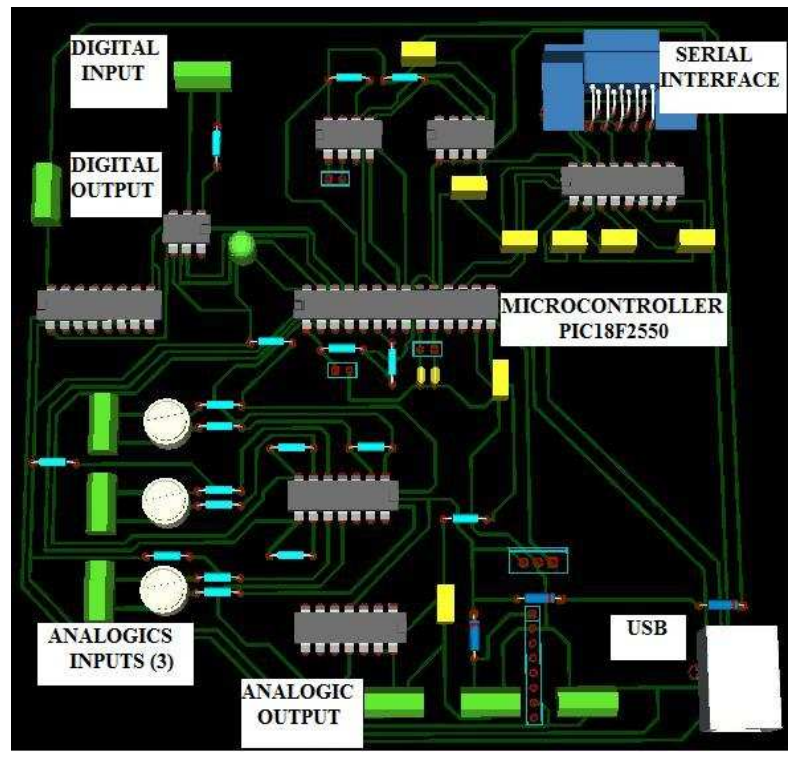

Fig. 1. The Controllogger board

The developed data acquisition system is a 10-bit system with 3 analog input channels designed for automatic longterm data collection. The LM324 operational amplifier was used for the analog signal amplification with a differential amplifier circuit configuration. Figure 2 shows the analog input circuit.

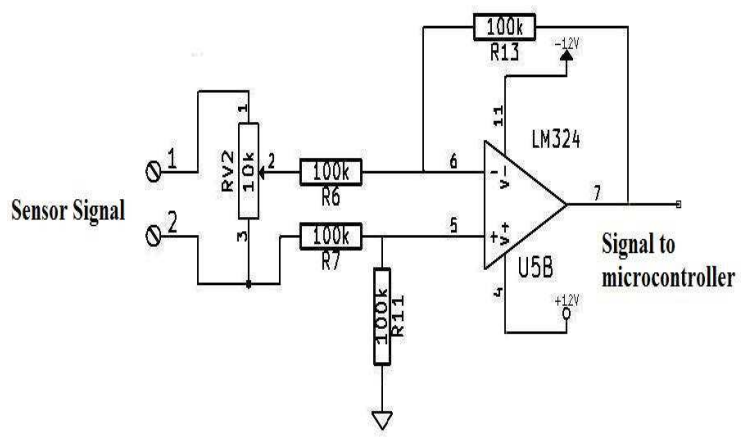

Fig. 2. Circuit for signal amplification

The main component of the data acquisition system is the PIC18F2550 microcontroller that is driven by a $20 \mathrm{MHz}$ crystal oscillator. An A/D converter of the microcontrollerbased unit records a set of sensor signals and the data are stored in a local EEPROM. The data collected by the microcontroller are transmitted to a GSM modem, with an RS-232 serial connection, being stored for further processing in a web server. Serial interfacing between the data acquisition system and the computer is implemented using the MAX232 line driver/receiver which is used to convert TTL $(0-5 \mathrm{~V})$ voltages required by the data acquisition system to the $10 \mathrm{~V}$ and $-10 \mathrm{~V}$ needed by the computer for RS232 communication. Figure 3 shows the serial connection from microcontroller to a GSM modem.

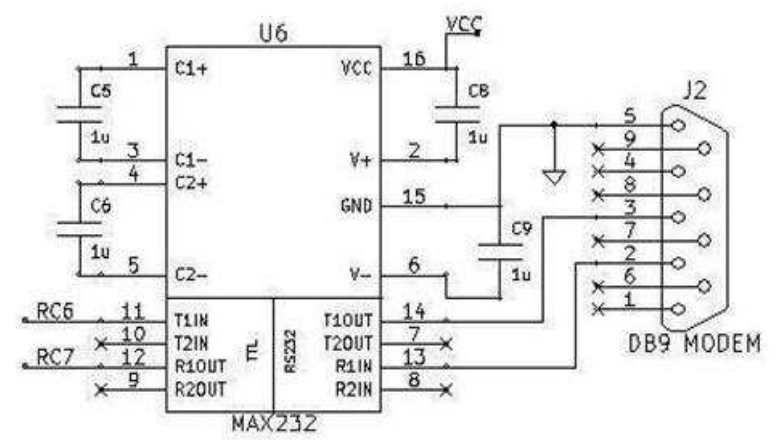

Fig. 3. RS232 connection in Controllogger board

\section{B. GSM Transmission}

The collected data are transmitted to a Database server by using a GSM modem. The data are received by a serial interface RS-232 from the Controllogger board and sent to a database server by a Java program. The GSM modem uses the GPRS connection to record the data in a database server. In order to send the information from the microcontroller to a GSM modem using the RS-232 interface, it is necessary to adapt the microcontroller output voltage to the GSM modem input voltage. A TTL/RS-232 transceiver module was used previously for this task.

\section{Web Monitoring}

The data visualization requires an internet connection to access the database. In this way, a developed web page permits the visualization of several characteristics from the decentralized renewable plant [4]. Figure 4 shows this interface, with the Weibull distribution of the collected wind speed values in a sampling day.

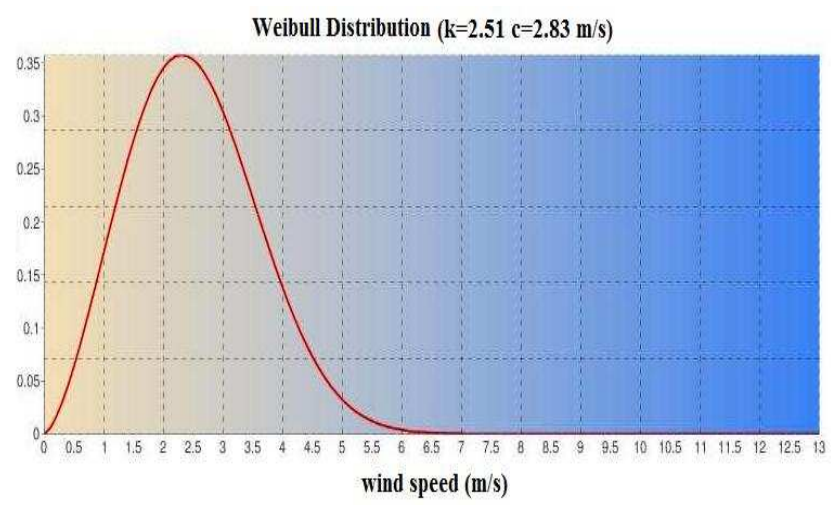

Fig. 4. Developed web page to show the Weibull distribution 
Figure 5 shows the collected wind speed values in a web page (24 hours).

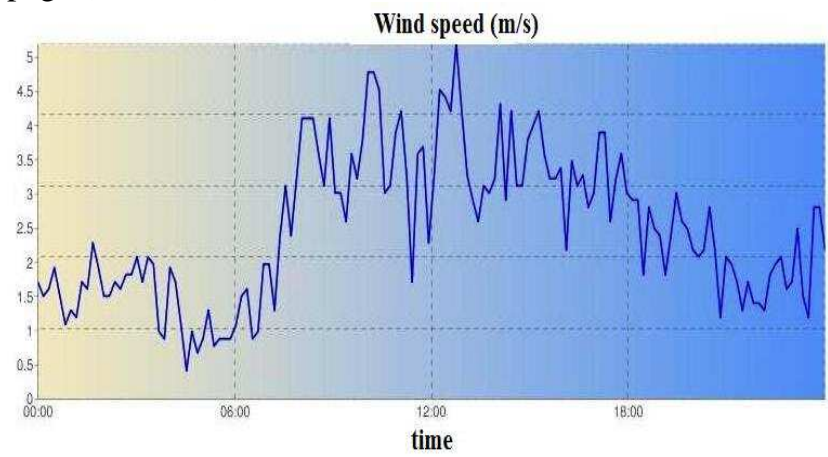

Fig. 5. Developed web page to show the collected wind speed (24 hours)

The PHP language is used to calculate the parameters $\mathrm{c}$ (scale parameter) and $\mathrm{k}$ (shape factor) of the Weibull distribution shown in figure 4 . The parameter $\mathrm{k}$ is calculated according to:

$$
k=\left(\frac{\sigma}{\bar{v}}\right)^{-1,086}
$$

The parameter $\mathrm{c}$ is calculated in (2):

$$
c=\frac{\bar{v}}{\Gamma\left(1+\frac{1}{k}\right)}
$$

Where the gama function $\Gamma$ is calculated according to:

$$
\Gamma(x)=\left[x e^{k_{E M} x} \prod_{n \in 1: \infty}\left(1+\frac{x}{n}\right) \cdot e^{\frac{-x}{n}}\right]^{-1}
$$

Figure 6 shows the general structure of the remote monitoring system applied to a wind speed sensor. The information from the anemometer goes to the microcontroller, where it is processed and sent to an external GSM modem. Therefore, the data are stored in a database server by the TCP/IP protocol.

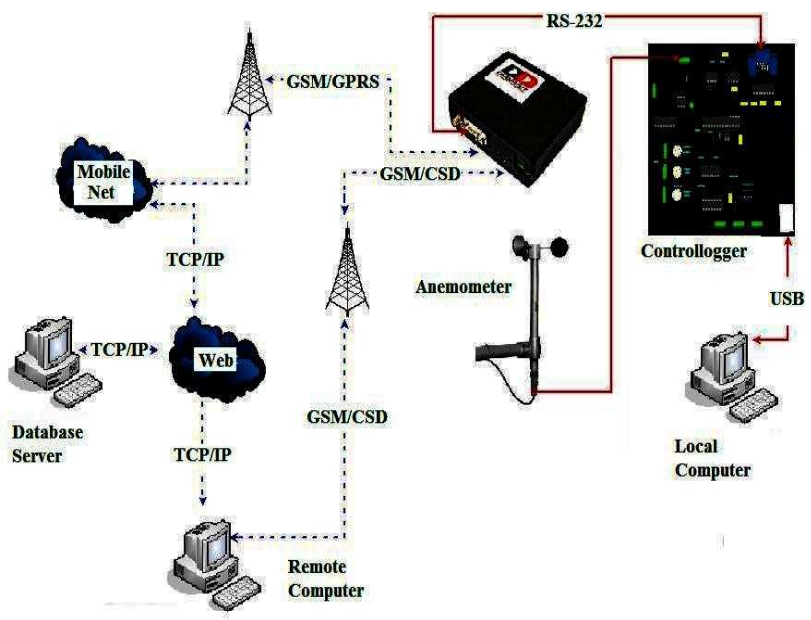

Fig. 6. General structure of the remote monitoring system
Figure 7 shows the used field data acquisition system (Controllogger) and the GSM modem.

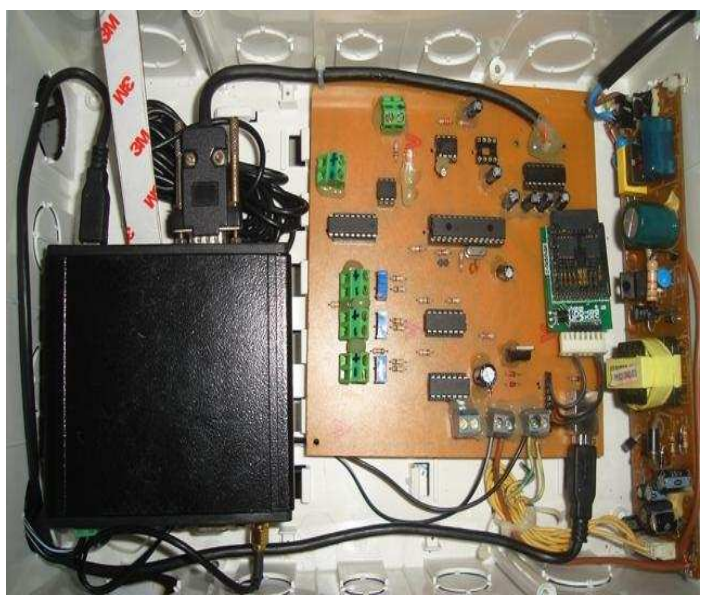

Fig. 7. The Controllogger and GSM modem

A memory card recorder (HDBS) is used to a local storing in the case of a data transmission failure caused by a GSM modem problem.

\section{Results}

The developed system was compared with a commercial data acquisition system. The commercial reference system was chose due to the high A/D converter resolution (13 bits). The results are given by using the Root Mean Square Error (RMSE), defined with:

$$
R M S E=\sqrt{\frac{\sum_{i=1}^{N}\left(x_{\text {ESTIMATED }}-x_{\text {MEASURED }}\right)^{2}}{N}}
$$

The results indicate that the Root Mean Square Error (RMSE) is $0.103 \mathrm{~m} / \mathrm{s}$, in the period 2011/04/02 2011/05/06 for a wind speed measurement tower at the Federal University of Ceará. Figure 8 shows results of 4,954 measurements from the used data acquisition systems (Controllogger and reference unity).

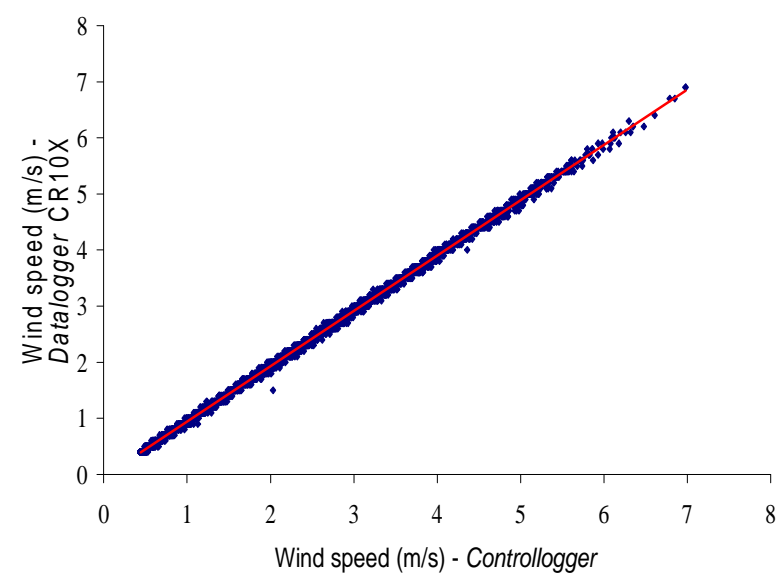

Fig. 8. Correlation between the wind speed measured by Controllogger and by the reference data logger 
According to Fig. 8, the measurements majority are located over the adjust line $1: 1$, illustrating the minimal dispersion of about 0.9996. Figure 9 shows wind speed measurements registered by Controllogger and by the reference data logger, in the period 2011/04/03 2011/04/05.

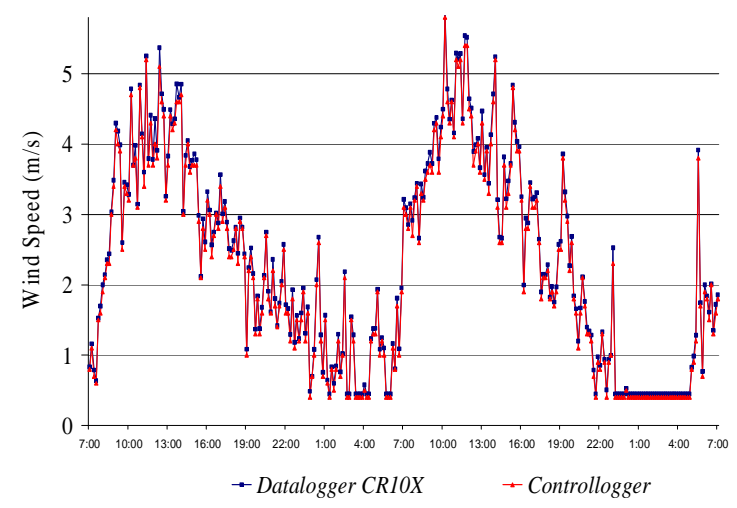

Fig. 9. Wind speed values measured by Controllogger and by the reference data logger

Figure 10 shows Weibull parameter c values based in the wind speed measured by the reference data logger and based in the PHP program, in the period December 2010 June 2011.

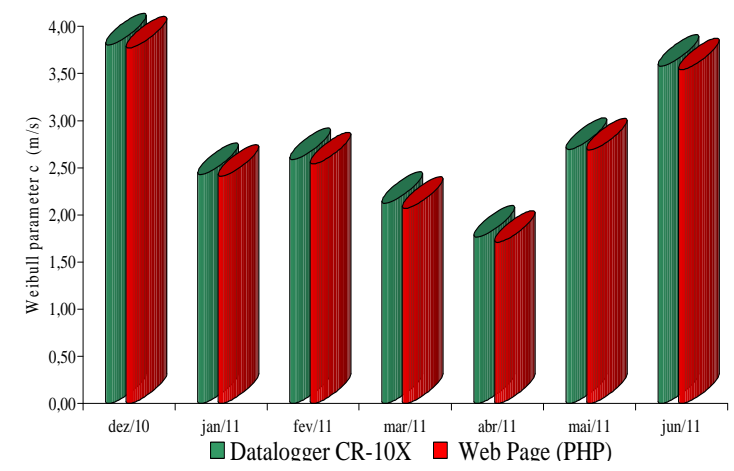

Fig. 10. Scale parameter $\mathrm{c}$ based in the reference data logger and in the PHP program.

Figure 11 shows Weibull parameter $\mathrm{k}$ values based in the wind speed measured by the reference data logger and based in the PHP program, in the period December 2010 June 2011.

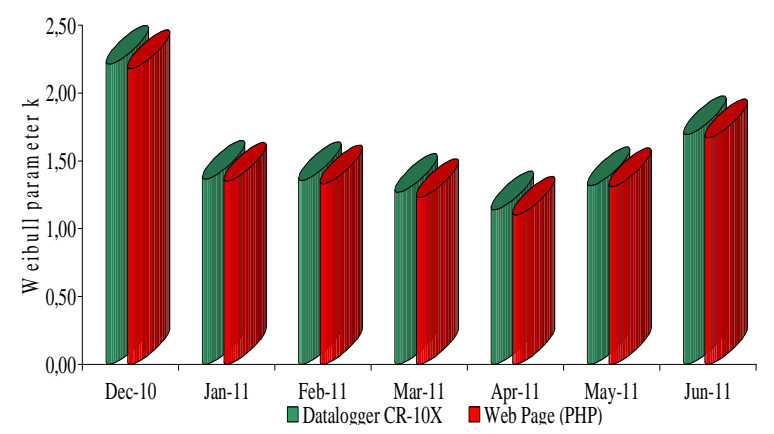

Fig. 11. Weibull parameter $\mathrm{k}$ based in the reference data logger and in the PHP program
Figure 12 shows average values based in the wind speed measured by the reference data logger and based in the PHP program, in the period December 2010 - June 2011.

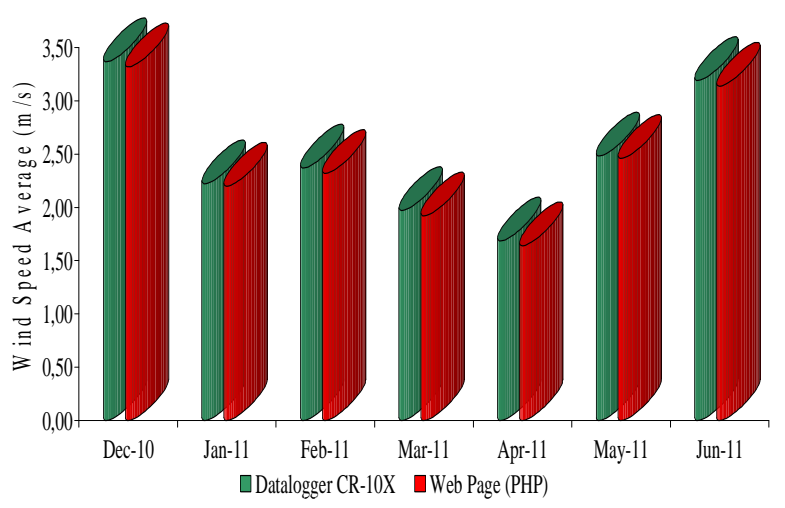

Fig. 12. Wind speed average based in the reference data logger and in the PHP program

\section{Conclusions}

The observed low errors indicate that the data have been stored in a similar form to what would be expected in a commercial data logger. This error rate suggests that the resolution of the data acquisition system is an essential influence factor. The A/D converter resolution of the used commercial equipment is 13 bit and the resolution of the developed system is 10 bit, due to the specified resolution of the used microcontroller.

It has been observed that the technology GSM/GPRS is the one that offers considerable advantages in transmitting the information at big distances. In the case of a data transmission failure caused by a GSM modem problem, the information is recorded in a memory card.

The use of the PHP and Java obtained satisfactory results to calculate the statistical parameters and to allow an improvement of the data transmission.

\section{Acknowledgements}

The authors would like to thank the financial support of $\mathrm{CNPq}$ for the present project.

\section{References}

[1] S.C.S. Jucá, P.C.M. Carvalho and F.T. Brito, "A low cost concept for data acquisition systems applied to decentralized renewable energy plants", Sensors, 2011, vol.11, pp. 743-756.

[2] M. Benghanem, "A low cost wireless data acquisition system for weather station monitoring", Renewable Energy, 2010, vol. 35, pp.862-872.

[3] S. Rosiek and F. Batlles, "A microcontroller-based dataacquisition system for meteorological station monitoring" Energy Conversion and Management, 2008, vol. 49, pp. 37463754.

[4] H. Belmili, S. Cheikh, M. Haddadi and C. Larbes, "Design and development of a data acquisition system for photovoltaic modules characterization”. Renewable Energy, 2010, vol. 35, pp.1484-1492. 